\title{
Hard-core repulsive interactions in even-parity electron pairings for heavy-fermion systems
}

\author{
F. C. Zhang \\ Theoretische Physik, Eidgenössische Technische Hochschule Zürich-Hönggerberg, 8093 Zürich, Switzerland \\ T. K. Lee \\ Department of Physics, Virginia Polytechnical Institute and State University, Blacksburg, Virginia 24061
}

(Received 9 December 1986)

\begin{abstract}
By studying the Anderson lattice Hamiltonian with spin-orbit coupling using an auxiliary boson method, we have examined the hard-core repulsive interactions in heavy-fermion materials. As a consequence of the anisotropy of the repulsive interaction, all lower-order partial-wave Cooper pairings in the even-parity channel are strongly impeded.
\end{abstract}

The recent discovery of heavy-fermion superconductors has aroused great interest among condensed-matter physicists. ${ }^{1,2}$ Although experiments have provided evidence that these superconductors are unconventional, the origin of the attractive interaction and the precise type of the Cooper pairing remain controversial. Both $p$-wave ${ }^{3-8}$ and $d$-wave ${ }^{9-13}$ Cooper pairings have been suggested. We note that in all the theoretically proposed $d$-wave pairings, ${ }^{9-12}$ the anisotropy of the hybridization between the conduction and the local $f$ states in heavy-fermion materials has not been taken into account. It is the purpose of this paper to examine the effect of this anisotropy on the possible Cooper pairings. We have studied the Anderson lattice model, taking into account the anisotropic hybridization, and we have found that the hard-core repulsive interaction of the quasiparticle Cooper pairs is anisotropic and of even parity. As a consequence, all the lower-order partial-wave Cooper pairings in even-parity channels are strongly impeded. The $d$-wave component of the repulsive interaction is found to be much larger than the recently reported weak attractive interaction due to the intersite scattering. ${ }^{9}$

The hard-core repulsive interaction in the Kondo problem was first studied by Nozières ${ }^{14}$ for a single-impurity Kondo Hamiltonian in the strong-coupling limit. The two electrons with opposite spins interact via a virtual process on the Kondo impurity, and the interaction is found to be repulsive. This conception has been confirmed recently to be also true for the Kondo lattice, where the interactions between two quasiparticles with different spins produced by scattering at the same Kondo ions is repulsive. $8,9,15,16$ In previous investigations, the hybridization between the conduction states and the Kondo ions or $f$ states is assumed to be isotropic for simplicity, such as in the $\operatorname{SU}(N)$ Anderson lattice model (where one assumes both the conduction electron and the $f$ electron have degeneracy $N$ ). The repulsive interaction in such cases is isotropic. This has been used to argue strongly against $s$-wave pairings in heavy-fermion superconductivity. The hybridization in real materials is, however, anisotropic. Therefore, the transformation from the original $f$-electron states to the quasiparticle states in heavy-fermion systems is anisotropic, the wave function of the quasiparticle depends strongly on the orientation of the particle momentum, and the hard-core repulsive interaction can have higher partial- wave components.

To examine the above idea quantitatively, we consider the Anderson lattice Hamiltonian

$$
\begin{aligned}
H= & \sum_{\mathbf{k}, \sigma} \varepsilon_{\mathbf{k}} C_{\mathbf{k} \sigma}^{\dagger} C_{\mathbf{k} \sigma}+\sum_{i, m} \varepsilon_{f} f_{i m}^{\dagger} f_{i m} \\
& +\frac{1}{\sqrt{N_{s}}} \sum_{\mathbf{k}, \sigma, m, i}\left(V_{\mathbf{k} \sigma m} e^{i \mathbf{k} \cdot \mathbf{R}_{i}} C_{\mathbf{k} \sigma}^{\dagger} f_{i m}+\text { H.c. }\right)
\end{aligned}
$$

with the constraint $\sum_{m} f_{i m}^{\dagger} f_{i m} \leq 1$ at each site.

In Eq. (1), the conduction electron is assumed to be a plane wave, and $m$ is the magnetic quantum index, running in the case of $\mathrm{Ce}^{3+}$ from $-\frac{5}{2}$ to $\frac{5}{2}$. The hybridization matrix element is assumed to have the form given by Coqblin and Schrieffer, ${ }^{17} V_{\mathbf{k} \sigma m}=(N / 2)^{1 / 2} V_{k} \beta_{m \sigma}(\hat{\mathbf{k}})$, where $N=2 J+1=6$, and

$$
\beta_{m \sigma}(\hat{\mathbf{k}})=-\left(\frac{4 \pi}{3}\right)^{1 / 2}(-i)^{3} \sigma\left(\frac{7-2 m \sigma}{14}\right)^{1 / 2} Y_{3, m-1 / 2 \sigma}^{*}(\hat{\mathbf{k}}) \text {. }
$$

For simplicity, we shall assume a constant density of states $\rho_{0}$ for the conduction electrons per spin, and assume $V_{k}$ to be a constant $V$.

We shall study the Hamiltonian using an auxiliary boson technique developed for the Kondo problem by Coleman ${ }^{18}$ and Read and Newns. ${ }^{19}$ The technique has been very recently applied to study the quasiparticle interactions in the $\mathrm{SU}(N)$ Anderson lattice. ${ }^{9,16,20}$ It is straightforward to apply the method to the present Hamiltonian. Let $r_{i}$ and $\theta_{i}$ be the amplitude and phase of the auxiliary Bose field describing an $f$ hole (empty $f$-electron state) at site $i$, and $\lambda_{i}=\tilde{\lambda}_{i}+\partial_{\tau} \theta_{i}$, with $\tilde{\lambda}_{i}$ the constraint scalar field. After taking a gauge transformation ${ }^{19} f_{i m} \rightarrow f_{i m} e^{i \theta_{i}}$, the system may be described in the functional integral form by the fermion fields and two scalar fields $r$ and $\lambda$.

We approximately describe the physical system in the mean-auxiliary-field theory and treat the fluctuation around the mean field as an interaction part. The mean values of the Bose fields are determined by a variational scheme. The solutions of the mean-field theory are regarded as the noninteracting system. Further, the interaction part is approximated by Gaussian fluctuations. Details are to be found in Refs. 9 and 19. 
The noninteracting fermion system in the functional intergral form is described by a single-particle Lagrangian. The resulting hybridized bands consist of twofold lower and upper extended states, and fourfold-degenerate localized $f$ bands with energy $T_{0}=\varepsilon_{f}-E_{0}$, with $E_{0}$ the mean value of the field $i \lambda . T_{0}$ is of the order of the Kondo temperature. The dispersions of the lower $(-)$ and upper $(+)$ bands are given by

$$
E_{\mathbf{k}}^{ \pm}=\frac{1}{2}\left[\varepsilon_{\mathbf{k}}+T_{0} \pm \sqrt{\left(\varepsilon_{\mathbf{k}}-T_{0}\right)^{2}+4 \alpha^{2}}\right] \text {, }
$$

where $\alpha^{2}=\left(T_{0}-\mu\right) / 2 \rho_{0}, \mu$ is the Fermi energy, and we assume $\mu \lesssim 0$ in this paper. Equation (3) is similar to that found by the Gutzwiller variational approach. ${ }^{21}$ The extended states are the combinations of conduction- and $f$ electron states:

$$
\begin{aligned}
& A_{+}(\mathbf{k} \sigma)=+\left(\cos \theta_{\mathbf{k}}\right) C_{\mathbf{k} \sigma}+\left(\sin \theta_{\mathbf{k}}\right) \sum_{m} \beta_{m \sigma}(\hat{\mathbf{k}}) f_{\mathbf{k} m}, \\
& A_{-}(\mathbf{k} \sigma)=\left(\sin \theta_{\mathbf{k}}\right) C_{\mathbf{k} \sigma}-\left(\cos \theta_{\mathbf{k}}\right) \sum_{m} \beta_{m \sigma}(\hat{\mathbf{k}}) f_{\mathbf{k} m},
\end{aligned}
$$

where $\theta_{\mathbf{k}}$ is defined by equation $\cot \theta_{\mathbf{k}}=\alpha /\left(T_{0}-E_{\mathbf{k}}^{-}\right)$. In Eqs. (4) we have used conduction-electron spin index $\sigma$ to denote the pseudospin of the quasiparticle because of the one-to-one correspondence between them. Equations (4) are identical to the results of the Korringa-Kohn-Rostoker equation approach, ${ }^{22,23}$ and the Green's-function method. ${ }^{24}$

The excitations of the noninteracting system are quasiparticles described by Eq. (4b) with energy close to $\mu$. The mass enhancement of quasiparticles is $m^{*} / m$ $=\left[2\left(T_{0}-\mu\right) \rho_{0}\right]^{-1} \gg 1$. Quasiparticles interact with each other due to the fluctuations of the auxiliary Bose fields. The boson propagator $D(\omega, \mathrm{q})$ is a $2 \times 2$ matrix corresponding to the fluctuation parts of the fields $r$ and $\lambda$. In the random-phase approximation,

$$
D^{-1}(\omega, \mathbf{q})=D_{0}^{-1}(\omega, \mathbf{q})-\Pi(\omega, \mathbf{q})
$$

where $D_{0}$ are the bare boson propagators given in the mean-field theory, and $\Pi$ are the boson self-energies given by various one-loop fermion diagrams. At zero frequency,

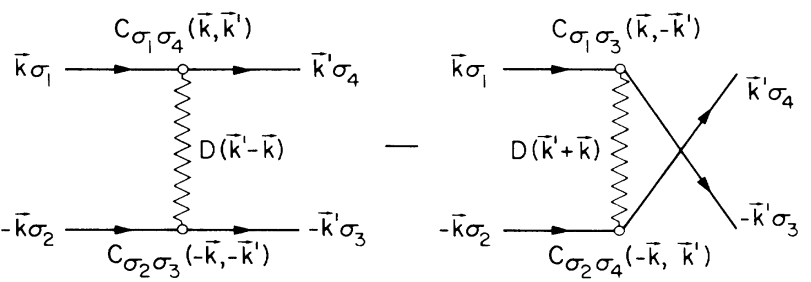

FIG. 1. Diagrammatic representation of the quasiparticle (solid lines) Cooper-pair vertex function, which involves an auxiliary boson exchange (wiggly lines). The $C$ 's are vertices, which are anisotropic, giving rise to the higher-order partial-wave components of the hard-core repulsive interaction.

we have found that

$$
\begin{aligned}
& D_{r r}^{-1}=2 N V^{2} \rho_{0} J_{1}, \\
& D_{r \lambda}^{-1}=-i \sqrt{2 N} V\left(1+\frac{1}{4} J_{2}\right) / \alpha=D_{\lambda r}^{-1}, \\
& D_{\lambda \lambda}^{-1}=-\left(T_{0}-\mu\right)^{-1}\left(1+\frac{1}{6} J_{3}\right),
\end{aligned}
$$

where $J_{n}$ is a function of $\mathbf{q}$, given by

$$
\begin{aligned}
J_{n}(\mathbf{q})=n \rho_{0}^{-2} \sum_{\mathbf{k}, \mathbf{k}^{\prime}} & \frac{f\left(E_{\mathbf{k}}^{-}\right)\left(T_{0}-\mu\right)^{n} \delta_{\mathbf{k}+\mathbf{q}, \mathbf{k}^{\prime}}+\mathbf{G}_{n}}{\left(E_{\mathbf{k}}^{+}-E_{\mathbf{k}}^{-}\right)\left(T_{0}-E_{\mathbf{k}}^{-}\right)^{n}\left(\varepsilon_{\mathbf{k}^{\prime}}-\varepsilon_{\mathbf{k}}\right)} \\
& \times \sum_{m, m^{\prime}, \sigma, \sigma^{\prime}} \beta_{m \sigma}(\hat{\mathbf{k}}) \beta_{m \sigma^{\prime}}^{*}\left(\hat{\mathbf{k}}^{\prime}\right) \beta_{m^{\prime} \sigma}^{*}(\hat{\mathbf{k}}) \beta_{m^{\prime} \sigma^{\prime}}\left(\hat{\mathbf{k}}^{\prime}\right) .
\end{aligned}
$$

In Eq. (7), $\mathbf{G}_{\boldsymbol{n}}$ is the reciprocal vector, $\mathbf{k}$ and $\mathbf{k}^{\prime}$ are within the first Brillouin zone. The angular part of the summation over $\mathbf{k}$ in Eq. (7) can be carried out for a quadratic bare conduction band, but the expression is lengthy. At the limit $q \rightarrow 0$, we have $J_{n}(q \rightarrow 0) \rightarrow \gamma / 2$, with $\gamma=\left(\rho_{0} k_{F / 2 m}^{2}\right)^{-1}$, and $k_{F}$ the Fermi momentum. $1<\gamma<2$, and $\gamma$ is close to 1 if $\mu \simeq 0$.

To study the superconductivity, we consider the quasiparticle-Cooper-pair scattering process represented diagrammatically in Fig. 1. We shall adopt the static approximation to calculate the vertex at the Fermi surface. We than have

$$
\Gamma_{\sigma_{1} \sigma_{2} \sigma_{3} \sigma_{4}}\left(\mathbf{k},-\mathbf{k} ;-\mathbf{k}^{\prime}, \mathbf{k}^{\prime}\right)=\sum_{i, j}\left[C_{\sigma_{1} \sigma_{4}}^{(i)}\left(\mathbf{k}, \mathbf{k}^{\prime}\right) C_{\sigma_{2} \sigma_{3}}^{(j)}\left(-\mathbf{k},-\mathbf{k}^{\prime}\right) D_{i j}\left(\mathbf{k}^{\prime}-\mathbf{k}\right)-C_{\sigma_{1} \sigma_{3}}^{(i)}\left(\mathbf{k},-\mathbf{k}^{\prime}\right) C_{\sigma_{2} \sigma_{4}}^{(j)}\left(-\mathbf{k}, \mathbf{k}^{\prime}\right) D_{i j}\left(\mathbf{k}^{\prime}+\mathbf{k}\right)\right]
$$

In Eq. (8), the first term is the direct interaction, and the second term is the exchange interaction. The $C$ 's are the vertices arising from the anisotropic hybridization of Eq. (2) and the resulted anisotropic orthogonal transformation of Eq. (4b). The indices $i$ and $j$ represent field $r$ or $\lambda$. The $C$ 's are given by

$$
\begin{aligned}
& C_{\sigma \sigma^{\prime}}^{(r)}\left(\mathbf{k}, \mathbf{k}^{\prime}\right)=-\sqrt{2 N} V_{k} \cos \theta_{k_{F}} \sin \theta_{k_{F}} \sum_{m} \beta_{m \sigma^{\prime}}^{*}\left(\hat{\mathbf{k}}^{\prime}\right) \beta_{m \sigma}(\hat{\mathbf{k}}), \\
& C_{\sigma \sigma^{\prime}}^{(\lambda)}\left(\mathbf{k}, \mathbf{k}^{\prime}\right)=i\left(\cos \theta_{k_{F}}\right)^{2} \sum_{m} \beta_{m \sigma}(\hat{\mathbf{k}}) \beta_{m \sigma^{\prime}}^{*}\left(\hat{\mathbf{k}}^{\prime}\right)
\end{aligned}
$$

The vertex of Eq. (8) can be decomposed into even-parity part and odd-parity part:

$\Gamma^{\text {even }}\left(\mathbf{k}, \mathbf{k}^{\prime}\right)=\frac{1}{2}\left[\Gamma\left(\mathbf{k},-\mathbf{k} ;-\mathbf{k}^{\prime}, \mathbf{k}^{\prime}\right)+\Gamma\left(\mathbf{k},-\mathbf{k} ; \mathbf{k}^{\prime},-\mathbf{k}^{\prime}\right)\right]$,

$\Gamma^{\text {odd }}\left(\mathbf{k}, \mathbf{k}^{\prime}\right)=\frac{1}{2}\left[\Gamma\left(\mathbf{k},-\mathbf{k} ;-\mathbf{k}^{\prime}, \mathbf{k}^{\prime}\right)-\Gamma\left(\mathbf{k},-\mathbf{k} ; \mathbf{k}^{\prime},-\mathbf{k}^{\prime}\right)\right]$

where the pseudospin indices are hidden.

The even-parity part of the vertex is the interaction between the pseudospin singlet Cooper pairs, where $\sigma_{1}+\sigma_{2}=\sigma_{3}+\sigma_{4}=0$. Under the random-phase approxi- 
mation, we obtain

$$
\begin{aligned}
\Gamma^{\text {even }}\left(\mathbf{k}, \mathbf{k}^{\prime}\right)= & \frac{1}{2}\left(T_{0}-\mu\right) W\left(\hat{\mathbf{k}}, \hat{\mathbf{k}}^{\prime}\right) \\
& \times\left(\frac{1}{1+S\left(\mathbf{k}^{\prime}-\mathbf{k}\right)}+\frac{1}{1+S\left(\mathbf{k}^{\prime}+\mathbf{k}\right)}\right),
\end{aligned}
$$

where

$$
S(\mathbf{q})=\frac{\frac{1}{6} J_{3}-\frac{1}{12} J_{1} J_{3}+\frac{1}{16}\left(J_{2}\right)^{2}}{1-\frac{1}{6} J_{3}+\frac{1}{2} J_{2}-\frac{1}{2} J_{1}}
$$

and $W\left(\hat{\mathbf{k}}, \hat{\mathbf{k}}^{\prime}\right)$ is the anisotropic angular function arising from the anisotropic hybridization

$$
W\left(\hat{\mathbf{k}}, \hat{\mathbf{k}}^{\prime}\right)=\frac{1}{2} \sum_{m, m^{\prime}, \sigma, \sigma^{\prime}} \beta_{m \sigma}(\hat{\mathbf{k}}) \beta_{m \sigma^{\prime}}^{*}\left(\hat{\mathbf{k}}^{\prime}\right) \beta_{m^{\prime} \sigma}^{*}(\hat{\mathbf{k}}) \beta_{m^{\prime} \sigma^{\prime}}\left(\hat{\mathbf{k}}^{\prime}\right)
$$

For our model, $W\left(\hat{\mathbf{k}}, \hat{\mathbf{k}}^{\prime}\right)$ is a function of $x=\hat{\mathbf{k}} \cdot \hat{\mathbf{k}}^{\prime}$ only, and

$$
W(x)=\frac{1}{3} P_{0}(x)+\frac{8}{21} P_{2}(x)+\frac{2}{7} P_{4}(x),
$$

with $P_{l}(x)$ the $l$ th Legendre polynomial.

To compare with the previous work, we note that for the isotropic hybridization, $W\left(\hat{\mathbf{k}} \cdot \hat{\mathbf{k}}^{\prime}\right)$ in Eq. (11) equals 1 . If we neglect function $S(\mathbf{q})$ for $\Gamma^{\text {even }}$ and consider the spin- $\frac{1}{2}$ case, namely, the orbital angular momentum of the localized electron being zero, Eq. (11) essentially recovers the hard-core repulsion obtained by Nozières. If we assume $\beta_{m \sigma}(\hat{\mathbf{k}})=\delta_{m \sigma}$, and let $\sigma$ have degeneracy $N$, we recover the result of the SU $(N)$ model of Lavagna, Millis, and Lee. ${ }^{9}$

$\Gamma^{\text {even }}$ in Eq. (11) may be separated into two parts. The first part is the value evaluated by setting $S(\mathbf{q})=0$, and the second part is the contribution from $S(q)$. The first part $\Gamma^{(1)}$ is independent of the momentum of the boson propagators as well as the lattice structure. It may be identified as the hard-core interaction of the Cooper pair. If we denote $\rho_{0}^{*}$ the quasiparticle density of states per pseudospin at the Fermi surface $\rho_{0}^{*}=\rho_{0} m^{*} / m$, then $\rho_{0}^{*} \Gamma_{\text {even }}^{(1)}=\frac{1}{2} W\left(\hat{\mathbf{k}} \cdot \hat{\mathbf{k}}^{\prime}\right)$. We arrive at the conclusion that the hard-core interactions on the all $l=0,2$, and 4 partial wave pairings $\Gamma_{l}^{(1)}$ are repulsive:

$$
\rho_{0}^{*} \Gamma_{0}^{(1)}=\frac{1}{6}, \rho_{0}^{*} \Gamma_{2}^{(1)}=\frac{4}{21}, \rho_{0}^{*} \Gamma_{4}^{(1)}=\frac{1}{7} .
$$

The vertex contributed from the momentum-dependent part of the boson propagators $\Gamma^{(2)}$ can be calculated numerically. In the case the bare conduction-electron dispersion is quadratic, we find ${ }^{25}$ that within the jellium approximation $\rho_{0}^{*} \Gamma^{(2)} \simeq-0.03 \gamma, \rho_{0}^{*} \Gamma_{2}^{(1)} \simeq-0.04 \gamma$, where $\gamma$ is order of 1 as we mentioned earlier. We see that the correction to the hard-core interaction from the fully randomphase approximation is about $20 \%$ only. Both $s$ - and $d$ wave pairings are not favored. The physical picture presented here is qualitatively different from that in the $\mathrm{SU}(N)$ model, where only $s$-wave pairing is suppressed.

We now study the odd-parity part of the vertex, which is the interaction between the triplet pseudospin Cooper pairs. We find that the hard-core interaction vanishes in the odd-parity channel. This is expected. The hybridization matrix $V_{\mathbf{k} \sigma m}$ has definite parity: $\boldsymbol{\beta}_{\boldsymbol{m} \sigma}(-\mathbf{k})$ $=-\beta_{m \sigma}(\mathbf{k})$. The product of the two vertices in the vertex of Eq. (8) always has even parity. The triplet pairing interaction is purely due to the $\mathbf{q}$ dependence of the boson propagators, and is given by

$$
\rho_{0}^{*} \Gamma_{\sigma_{1} \sigma_{2} \sigma_{3} \sigma_{4}}^{\text {odd }}\left(k, k^{\prime}\right)=\frac{1}{2}\left(\frac{1}{1+S\left(\mathbf{k}^{\prime}-\mathbf{k}\right)}-\frac{1}{1+S\left(\mathbf{k}^{\prime}+\mathbf{k}\right)}\right) \sum_{m, m^{\prime}} \beta_{m \sigma_{1}}(\hat{\mathbf{k}}) \beta_{m^{\prime} \sigma_{2}}(\hat{\mathbf{k}}) \cdot\left[\beta_{m \sigma_{4}}^{*}\left(\hat{\mathbf{k}}^{\prime}\right) \beta_{m^{\prime} \sigma_{3}}^{*}\left(\hat{\mathbf{k}}^{\prime}\right)+\beta_{m \sigma_{3}}^{*}\left(\hat{\mathbf{k}}^{\prime}\right) \beta_{m^{\prime} \sigma_{4}}^{*}\left(\hat{\mathbf{k}}^{\prime}\right)\right]
$$

The total pseudospin $z$ component of the triplet Cooper pair is not conserved because of spin-orbit coupling. Equation (15) provides a microscopic basis to study the triplet superconductivity and the Fermi liquid theory in the presence of spin-orbit coupling. Some parametrization schemes on this subject have been forwarded by Ueda and Rice, ${ }^{26}$ and Monier, Scharnberg, Tewordt, and Schopohl. ${ }^{27}$ We shall leave these for future work.

We have also used Goldstone-Feynman diagrammatic technique ${ }^{8,28}$ to calculate the hard-core interaction for Hamiltonian (1), and the identical results are obtained. We note that the anisotropic function $W(x)$ enters into the logarithmic divergent part interaction when one considers the ladder diagrams of Cooper-pair scatterings. Therefore this anisotropy truly affects the Cooper pairings.

In conclusion, we have studied the Anderson lattice model including spin-orbit coupling and anisotropic hybridization using the auxiliary boson method. The quasiparticle-Cooper-pair scattering vertex shows strong hard-core repulsive interactions on all $s$ wave, $d$ wave, and $l=4$ partial waves in even-parity channel. We like to emphasize that $d$-wave pairing is as unfavorable as $s$ wave. The hard-core vertex function may correspond to the contact interaction in spin-fluctuation model for ${ }^{3} \mathrm{He}$. The an- isotropic hard-core vertex therefore suggests that an appropriate spin-fluctuation model for the heavy-fermion materials should take into account the momentum dependence of the contact interaction, especially for the purpose of studying superconductivity. We note that the meanfield solutions of Hamiltonian (1) are not the exact solutions in the large-degeneragy $(N)$ limit. We may regard the mean-field theory and the random-phase approximation as an approach independent of $1 / N$ expansion.

Although the quantitative results presented in this paper apply to the symmetric $J=\frac{5}{2}$ case, we believe the qualitative results examined in this paper should apply to all heavy-fermion systems exhibiting Kondo effect. The anisotropy in hybridization is a general feature in Ce and $U$ compounds, the suppression of Cooper pairings in evenparity channel is a consequence of this anisotropy. This makes both $s$ - and $d$-wave pairing unlikely in heavyfermion superconductivity.

One of us (F.C.Z.) wishes to express his deep gratitude to T. M. Rice for his inspiration and many useful discussions. The work has been partly supported by the Swiss National Science Foundation. 
${ }^{1}$ P. A. Lee, T. M. Rice, J. W. Serene, L. J. Sham, and J. W. Wilkins, Comments Condens. Matter Phys. 12, 99 (1986).

${ }^{2}$ G. R. Stewart, Rev. Mod. Phys. 56, 755 (1984).

${ }^{3}$ P. W. Anderson, Phys. Rev. B 30, 1549 (1984); 30, 4000 (1984).

${ }^{4}$ H. R. Ott, H. Rudigier, T. M. Rice, K. Ueda, Z. Fisk, and J. L. Smith, Phys. Rev. Lett. 52, 1915 (1984).

${ }^{5}$ C. M. Varma, Bull. Am. Phys. Soc. 29, 404 (1984).

${ }^{6}$ O. T. Valls and Z. Tesanovic, Phys. Rev. Lett. 53, 1497 (1984).

${ }^{7}$ C. J. Pethick, D. Pines, K. F. Quader, K. S. Bedell, and G. E. Brown, Phys. Rev. Lett. 57, 1955 (1986).

${ }^{8}$ F. C. Zhang, T. K. Lee, and Z. B. Su, Phys. Rev. B (to be published).

${ }^{9}$ M. Lavagna, A. J. Millis, and P. A. Lee, Phys. Rev. Lett. 58 266 (1987).

${ }^{10}$ K. Miyake, S. Schmitt-Rink, and C. M. Varma, Phys. Rev. B 34, 6554 (1986).

${ }^{11}$ D. J. Scalapino, E. Loh, and J. E. Hirsch, Phys. Rev. B 34, 8190 (1986).

${ }^{12} \mathrm{M}$. Cyrot (unpublished).

${ }^{13}$ The possibility of $d$-wave pairing has also been studied by M. T. Béal-Monod, C. Bourbonnais, and V. J. Emery (unpublished).
${ }^{14}$ P. Nozières, J. Low Temp. Phys. 17, 31 (1974).

${ }^{15}$ T. K. Lee, J. Phys. C 18, L31 (1985).

${ }^{16}$ A. Auerbach and K. Levin, Phys. Rev. Lett. 57, 877 (1986).

${ }^{17}$ B. Coqblin and J. R. Schrieffer, Phys. Rev. 185, 847 (1969).

${ }^{18}$ P. Coleman, Phys. Rev. B 29, 3035 (1984).

${ }^{19}$ N. Read and D. Newns, J. Phys. C 16, 3273 (1983); Solid State Commun. 52, 993 (1984).

${ }^{20}$ A. J. Millis and P. A. Lee (unpublished).

${ }^{21}$ T. M. Rice and K. Ueda, Phys. Rev. Lett. 55, 995 (1985); 55, 2093(E) (1985).

${ }^{22}$ Z. Zou and P. W. Anderson, Phys. Rev. Lett. 57, 2073 (1986).

${ }^{23}$ H. Razafimandimby, P. Fulde, and J. Keller, Z. Phys. B 54, 111 (1984).

${ }^{24}$ T. K. Lee and F. C. Zhang, Phys. Rev. B 34, 8114 (1986).

${ }^{25}$ In numerical calculations, we have approximated $J_{2}$ and $J_{3}$ by $J_{1}$.

${ }^{26}$ K. Ueda and T. M. Rice, Phys. Rev. B 31, 7114 (1985).

${ }^{27}$ H. Monier, K. Scharnberg, L. Tewordt, and N. Schopohl, J. Low Temp. Phys. (to be published).

${ }^{28}$ N. Grewe and H. Keiter, Phys. Rev. B 24, 4420 (1981). 